\title{
High expression of Myosin 1g in pediatric acute lymphoblastic leukemia
}

\author{
Laura A. Estrada-Abreo ${ }^{1,2}$, Leonor Rodríguez-Cruz², Yanelly Garfias-Gómez ${ }^{1}$, \\ Janeth E. Araujo-Cardenas ${ }^{1}$, Gabriela Antonio-Andrés ${ }^{3}$, Alfonso R. Salgado- \\ Aguayo $^{4}$, Darío Orozco-Ruiz ${ }^{5}$, José Refugio Torres-Nava5 ${ }^{5}$ Juan D. Díaz-Valencia ${ }^{1}$, \\ Sara Huerta-Yépez ${ }^{3}$ and Genaro Patiño-López ${ }^{1}$ \\ ${ }^{1}$ Immunology and Proteomics Laboratory, Hospital Infantil de México Federico Gómez, México City, México \\ ${ }^{2}$ Cell Biology and Flow Cytometry Laboratory, Department of Health Sciences, Universidad Autónoma Metropolitana, \\ Iztapalapa, México \\ ${ }^{3}$ Oncologic Diseases Research Unit, Hospital Infantil de México Federico Gómez, México City, México \\ ${ }^{4}$ Laboratory of Research on Rheumatic Diseases, National Institute of Respiratory Diseases, Ismael Cosío Villegas, México \\ City, México \\ ${ }^{5}$ Oncology Service, Hospital Infantil Moctezuma, México City, México \\ Correspondence to: Genaro Patiño-López, email: gpatino@himfg.edu.mx
}

Keywords: Myosin 1g; acute lymphoblastic leukemia; high risk; biomarker; pediatric

Received: March 13, $2021 \quad$ Accepted: August 13, $2021 \quad$ Published: September 14, 2021

Copyright: ( $) 2021$ Estrada-Abreo et al. This is an open access article distributed under the terms of the Creative Commons Attribution License (CC BY 3.0), which permits unrestricted use, distribution, and reproduction in any medium, provided the original author and source are credited.

\section{ABSTRACT}

Acute Lymphoblastic Leukemia (ALL) is the most frequent cancer in pediatric population. Although the treatment has improved and almost $85 \%$ of the children are cured about $20 \%$ suffer relapse, therefore finding molecules that participate in the pathogenesis of the disease for the identification of relapse and patients at risk is an urgent unmet need. Class I myosins are molecular motors involved in membrane tension, endocytosis, phagocytosis and cell migration and recently they have been shown important for development and aggressiveness of diverse cancer types, however Myo1g an hematopoietic specific myosin has not been studied in cancer so far. We evaluated the expression of Myo1g by qRT-PCR, Immunocytochemistry and Immunofluorescence in a cohort of 133 ALL patients and correlated the expression at diagnosis and after treatment with clinical features and treatment outcomes. We found high expression levels of Myo1g in Peripheral Blood Mononuclear Cells (PBMCs) from patients with ALL at diagnosis and those levels decreased after complete remission; furthermore, we found an increase in Myo1g expression on patients with 9:22 translocation and those who relapse. This study show that Myo1g is over expressed in ALL and that may participate in the pathogenesis of the disease specially in high-risk patients.

\section{INTRODUCTION}

Acute Leukemias constitute a heterogeneous group of malignant neoplasms characterized by the clonal proliferation of hematopoietic precursors within the bone marrow; they are originated from a malignant transformation of lymphoid or myeloid progenitor cells [1]. Leukemia is the most common type of cancer in childhood, particularly affects children under 15 years old [2], with a prevalence between the ages of 2 to 5 years.
Acute Lymphoblastic Leukemia (ALL) corresponds to $78 \%$ of the cancer cases diagnosed in the pediatric population [3]. This neoplasm is classified into B-cell precursors (B-ALL) and T-cell precursors (T-ALL); the immunophenotype of B-cell precursors represents nearly $85 \%$ of the leukemia cases with the remaining $15 \%$ show a T-cell phenotype [4, 5]. Risk stratification of patients is an important component of diagnosis because it can help to decide the appropriate chemotherapy treatment and although current treatments have led to a general cure rate 
of more than $80 \%$ in children, however 15 to $20 \%$ of them suffer relapse. Some of the known high-risk factors for relapse are: age less than a year and older than 10 years, CNS infiltration at the time of diagnosis, T-cell phenotype, 9:22 translocation and lack of response to remission. Most relapses occur in bone marrow, however, approximately $33 \%$ of the cases have relapse to the Central Nervous System (CNS) and 7\% to testicles, why those sites are the target of relapse is unknown however, an hypothesis is that the leukemic cells gain the ability to produce colonies at a distance, using a variety of migration modes to achieve a successful invasion; this cellular movement depends on structural changes at the cytoskeleton level and the participation of motor proteins, chemokines and adhesion molecules [6-9].

Class I myosins are a family of actin dependent molecular motors involved in different functions like endocytosis, exocytosis, vesicle trafficking and different aspects of cell migration [10,11], all class I myosins associate to cell membranes through the tail domain that contains a lipid binding domain helping to fulfill their functions including generating movement in the cell [12, 13], Humans have eight genes that code for these proteins Myola-Myo1h and are subdivided in short tail (Myola, b, c, d, g, h) and long tail (Myole, f) [11], Myolg is exclusively expressed in hematopoietic cells and is highly expressed in $\mathrm{B}$ and $\mathrm{T}$ lymphocytes $[14,15]$. We and others have reported that Myolg plays an important role in membrane tension, cellular rigidity and regulation of the intrinsic velocity of the cell. It has also been reported that the deficiency of this protein produces changes in structures such as filopodia and microvilli [15-19]. And recently class I myosins have been gained interest in the cancer field because some of them function as tumor suppressors and some others are over expressed in different cancers [20-22], however so far there is no indication of the direct involvement of Myolg in cancer, therefore the aim of this study was to determine the expression levels of Myosin $1 \mathrm{~g}$ in blood cells of patients with acute lymphoblastic leukemia and to correlate this expression with the severity of the disease and with treatment outcomes in children with ALL.

\section{RESULTS}

\section{Myosin 1g is over expressed in acute lymphoblastic leukemia}

Myosins have various functions in different cell lineages $[10,12]$ and recently their study has gained interest in cancer research [23-27]. To determine the expression level of Myo1g in ALL, we initially quantified the mRNA expression of Myolg in 9 samples of peripheral blood and 9 samples of bone marrow from the same patients, subsequently we analyzed peripheral blood samples from patients, we follow them during the course of the treatment (when possible), specifically we analyzed 102 patients at diagnostic, 70 at remission and 73 at consolidation and compared those levels with 17 pediatric controls by real time PCR, the expression of GAPDH was used as internal control. We observed upregulation of Myo1g in all phases of the disease, being more significant at diagnostic with a trend to diminish at consolidation $\left({ }^{* *} P<0.01\right.$ diagnostic, ${ }^{*} P<0.05$ at remission and NS at consolidation) Figure $1 \mathrm{~A}$. Moreover we explored the expression of all 8 members of the class I myosin subfamily to determine which of them are upregulated in pediatric ALL, we compared levels of these Myosins in 10 hematopoietic normal pediatric individuals, 10 healthy adults and 10 patient PBMCs and found that Myolb and Myolg were the two Myosins more upregulated in patients Figure 1B, interestingly we found differences in myosin I expression between pediatric and adult controls, indicating that the best comparison should be pediatric individuals. We also evaluated expression of Myolg in different cell lines, we found that Myolg was consistently over expressed in a B-ALL cell line (RS4:11) Figure 1C. Interestingly when we classified patients according to the risk, we found that myolg was upregulated in highrisk patients $\left({ }^{* * *} P<0.001\right.$, and was Not Significant $p=$ 0.06 in Standard risk patients) Figure 1D, indicating that Myolg could be associated with high-risk patients of pediatric ALL. To determine whether Myo1g expression could predict patients with ALL versus normal controls we generated ROC curves, we found AUC value of 0.78 , $P<0.001$ for high-risk Figure $1 \mathrm{E}$ and $0.75, P<0.01$ for standard risk patients Figure 1F, using the expression of Myo1g in the control group as reference. Taken together these data indicated that Myolg expression is increased in ALL specially in high-risk patients.

\section{Myosin 1g over expression has diagnostic value in ALL}

To determine the clinical significance of Myolg over expression in ALL with other clinical parameters we evaluated our cohort of patients and followed the expression of Myolg over the treatment at the remission phase and at consolidation comparing different outcomes, for this we evaluated Myo1g expression levels in PBMCs from the patients who live and died and scored the Fold Change of Myolg on those cells. We found that patients at diagnosis have higher expression of Myolg compared to controls ${ }^{* *} P<0.01$, interestingly Myolg expression have a trend to decrease after treatment returning to levels similar to those of the controls ${ }^{*} P<0.05$ Figure $2 \mathrm{~A}$. Unexpectedly we didn't find obvious over expression of those patients who died at the different phases of treatment Figure 2A. To our knowledge Myo1g has not been studied as a marker in other diseases; however, TCGA data from Renal Cancer patients (857 cases) showed significant less expression of Myolg in those patients who survived versus those who 
$\operatorname{died}^{* * * *} P<0.0001$ Figure $2 \mathrm{~B}$. Using the same dataset from TCGA we evaluated the prognostic value of Myolg in the renal cancer patients and found a significant difference $(p$ $<0.0001$ ) indicating that high Myolg expression correlate with poor survival Figure 2C. However, when we analyzed the prognostic value of Myolg in our data we didn't find significant differences (not shown) this maybe is caused by the low patient number in our cohort, further studies might test this possibility. ALL therapy has improved in the recent years however, still there are $10-15 \%$ of patients who relapse and those almost invariably will die within few years, therefore a way to identify early on those patients at risk would improve their outcome, increasing their life expectancy and event free survival, along this line we decided to evaluate if there was a correlation between Myolg expression and patient relapse, we found that compared to controls the patients who relapse expressed higher levels of Myo1g at diagnostic ${ }^{*} P<0.05$, and at remission ${ }^{* *} P<0.01$ but not significant difference at consolidation Figure 2D, interestingly data from a published dataset (GSE13576) which, provide data for relapse in leukemia patients showed statistical significance in the expression of Myolg on those patients who had early relapse $(P=0.0008)$ Figure $2 \mathrm{E}$. To determine the diagnostic value of Myolg with other high risk clinical parameters we compared the expression of Myolg in controls with patients (Supplementary Figure 1) and generated ROC curves, we found differences for patients with poor prednisone response (PPR) ${ }^{* *} P=0.0017$, AUC $0.792, P<0.082$, Patients who have no remission ${ }^{* *} P=$ 0.0016 , AUC 0.812, $P<0.001$, Patients with infiltration to $\mathrm{SNC} \mathrm{P}=\mathrm{NS}$, AUC $0.676, P<0.086$ and interestingly in patients with translocation $\mathrm{t}(9 ; 22)$ at diagnostic $P<0.05$, AUC 0.889, $P<0.017$ (Supplementary Figure 1). Taken together our results indicate that Myolg could be used as a potential diagnostic marker, further studies will be designed to analyze this possibility. Immunocytochemistry (ICQ) is a technique of routine used in pathology laboratories in diseases such as leukemia, therefore we evaluated if Myo1g behaves similarly by ICQ and Immunofluorescence as does for mRNA expression, in normal cells Myolg is mainly expressed at the plasma membrane $[14,15]$, when we evaluated Myo1g expression in the same cohort of patients, we found a significant difference between healthy controls and patients in all phases of treatment Figure 3, we observed an increased Myolg expression by ICQ at diagnosis and consolidation ${ }^{*} P<0.05$ Figure $3 \mathrm{~A}$, by this technique we detected membrane and cytoplasmic signal Figure 3B, again we evaluated correlation of Myolg expression with high
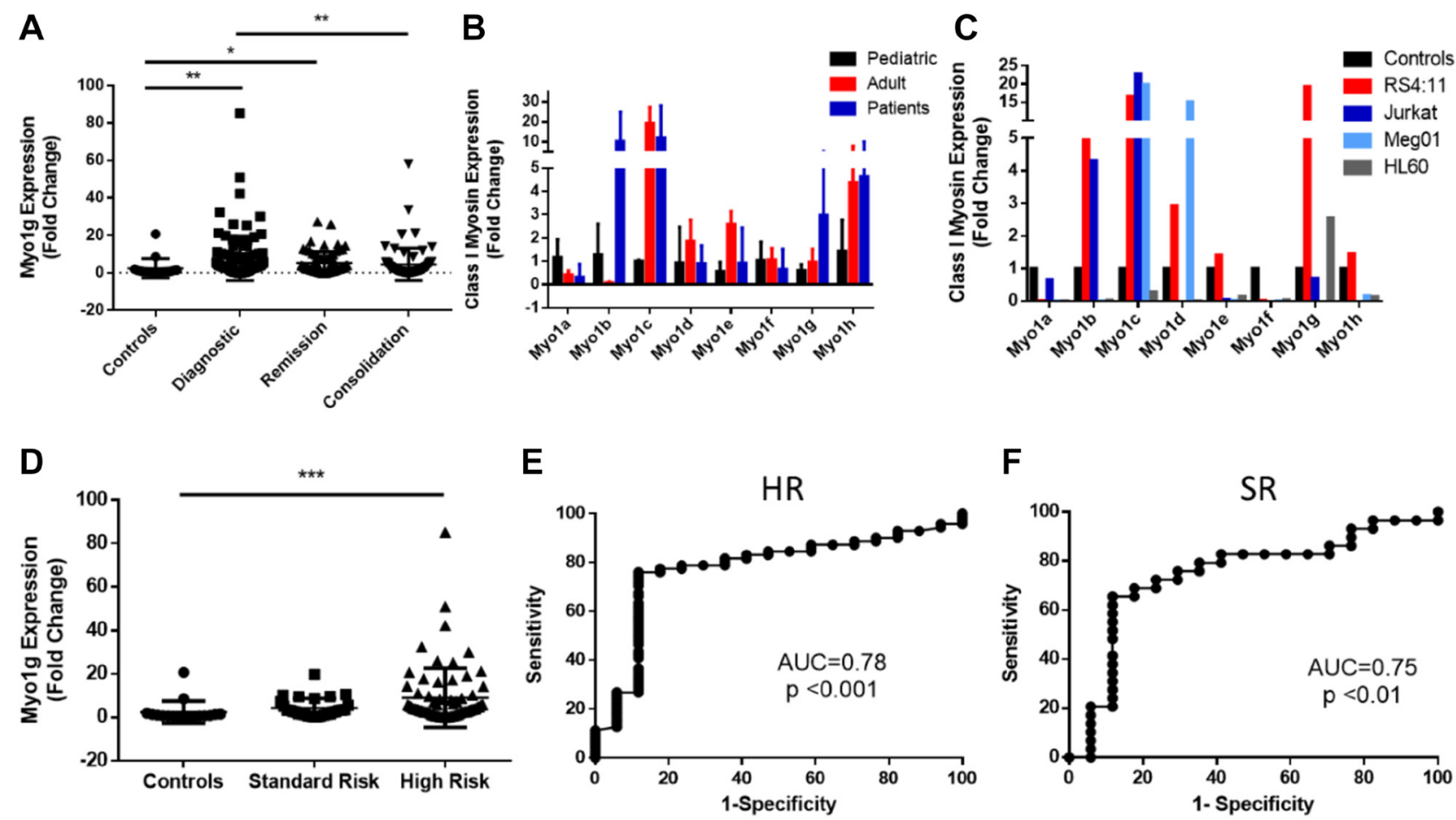

Figure 1: Myosin 1g is over expressed in acute lymphoblastic leukemia. (A) Fold Change of Myo1g mRNA expression in ALL pediatric patients (diagnostic $n=100$, remission $n=68$, consolidation $n=72$ ) and healthy controls $n=17$ ). One Way ANOVA with Dunn's correction, Error bars represent standard deviation, ${ }^{*} P<0.05,{ }^{* *} P<0.01$. (B) Class I myosin expression in pediatric $n=3$ and adult $n=3$ control PBMC cells compared to PBMCs from ALL patients $n=10$. Error bars represent standard deviation. (C) Class I myosin expression in B-ALL (RS4;11), T-ALL (Jurkat), CML (Meg-01) and APL (HL-60) cell lines representative result from an experiment done in triplicates, (D) Myolg expression in standard $n=19$ and high-risk patients $n=71$ compared to the expression in control individuals $n$ $=17$. $(\mathbf{E})$ ROC curves with corresponding AUC values for high-risk patients and (F) standard risk patients using the level of expression in control individuals as reference. 
risk clinical parameters by ICQ compared to controls, we found ${ }^{* *} P<0.01$ in patients with high risk and $p<0.05$ in patients with no remission (Supplementary Table 1), unexpectedly we did not found significant difference in Myo1g expression by immunofluorescence Figure 3C, although, we observed plasma membrane staining in normal and leukemic cells and found that leukemic cells often expressed elevated levels also at the cytoplasm and those levels continued higher after treatment Figure 3D. and evaluating Myolg expression with high risk clinical parameters we found increased expression in patients with high risk ${ }^{* * *} P<0.001$, patients with Translocation $\mathrm{t}(9: 22)$ ${ }^{* *} p<0.01$, patients with infiltration to $\mathrm{CNS}^{* * *} P<0.001$ and patients with no remission ${ }^{*} P<0.05$ (Supplementary Table 2). Overall, our results show that Myolg is over expressed in ALL patients and continue over expressed even early at consolidation, however, we found a trend towards basal levels in those patients who are responding well to the treatment.

\section{DISCUSSION}

Acute Lymphoblastic Leukemia is the cancer with the highest incidence in children worldwide, although the cure rate has increased with current therapies; we still find about $20 \%$ of patients who relapse and others who do not respond to treatment. Therefore, it is necessary to identify molecules that help to better diagnose the patients, molecules that predict the course of the disease and the stratification of risk to improve the outcomes and to give more tailored treatment to the patients $[28,29]$. Class I myosins recently have gained interest as potential biomarkers for different cancer types and for their participation in the development of the disease [24-26, 30]. We evaluated the expression of Myo1g an hematopoietic restricted class I myosin that has not been studied in cancer before by qPCR, Immunofluorescence and ICQ at diagnosis, after complete remission and early in consolidation. We found that Myolg is over
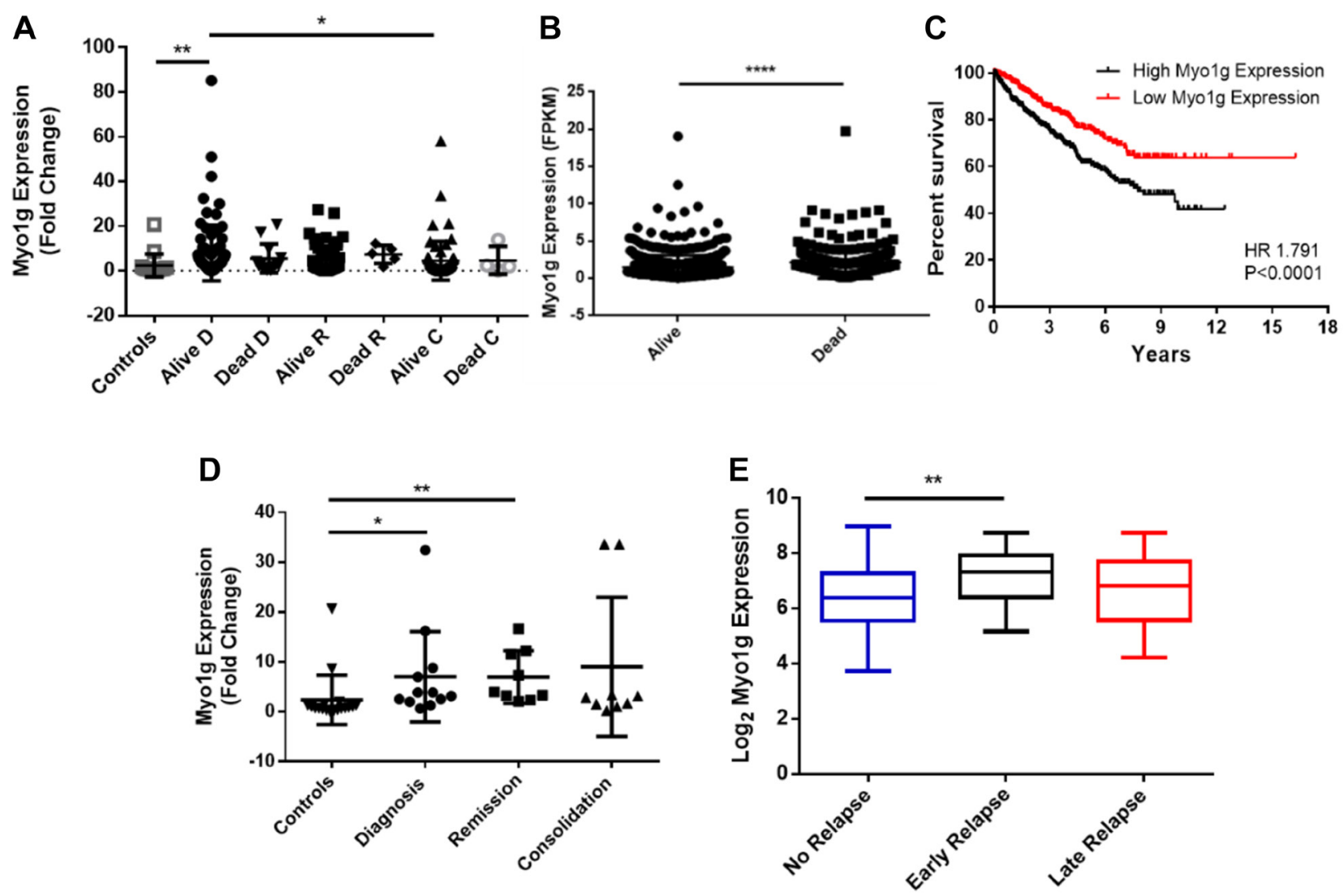

Figure 2: Myo1g is a potential biomarker in pediatric acute lymphoblastic leukemia. (A) Fold change of Myo1g expression in peripheral blood mononuclear cells (PBMC) of ALL pediatric patients alive $n=87$ or dead $n=13$ after diagnosis, alive $n=63$, dead $n=$ 5 at remission and alive $n=68$, dead $n=4$ at consolidation and controls $n=17$. Error bars represent mean $+/-\mathrm{SD}$, One-way ANOVA with Dunn's correction ${ }^{*} P<0.05,{ }^{* *} P<0.01$. (B) mRNA expression levels of Myo1g from TCGA data from Liver cancer patients alive $n=651$ and death $n=226$. Unpaired $t$ test, $P<0.0001$. (C) Kaplan-Meier curve of overall survival of Myo1g mRNA expression data from TCGA renal cancer data containing 877 patients, the median of Log 2 expression was used to define low $(n=438)$ and high $(n=439)$ expression (D) Fold Change of Myo1g expression in PBMCs from patients who relapse after Diagnostic $n=12$, at remission $n=9$ and at consolidation $n=9$ compared to controls $n=18$. Error bars represent mean $+/-\mathrm{SD}$, One-way ANOVA with Dunn's correction, ${ }^{* *} P<0.01,{ }^{*} P<0.05$. $(\mathbf{E})$ mRNA expression of Myo1g in patients with no relapse $n=157$, early relapse $n=26$ and late relapse $n=14$, data from GEO GSE13576, One Way $\operatorname{ANOVA}^{* *} P<0.01$ 
expressed at mRNA and to some extent at protein level in PBMCs from ALL patients and identified Myo1g as a molecule that participates in the course of the disease, we suggest that it is involved in the pathogenesis of the pediatric ALL. Although was more significant for HighRisk patients, we also detected differences in patients with infiltrations, those who suffer relapse and for those patients at high risk of death. Importantly, we used data from a previous study that used microarrays to evaluate expression of differential genes in patients at relapse, we found a statistically significant difference $P<0.05$ between those patients who suffer early relapse versus those with no relapse on the expression of Myolg [28] consistent with our results, from those patients who relapsed at diagnostic and at consolidation, however we need more patients and longer follow up to determine the predictive value of Myolg at relapse. Myolg expression levels were considerably higher ${ }^{*} P=0.0177$ and ${ }^{* *} P<$

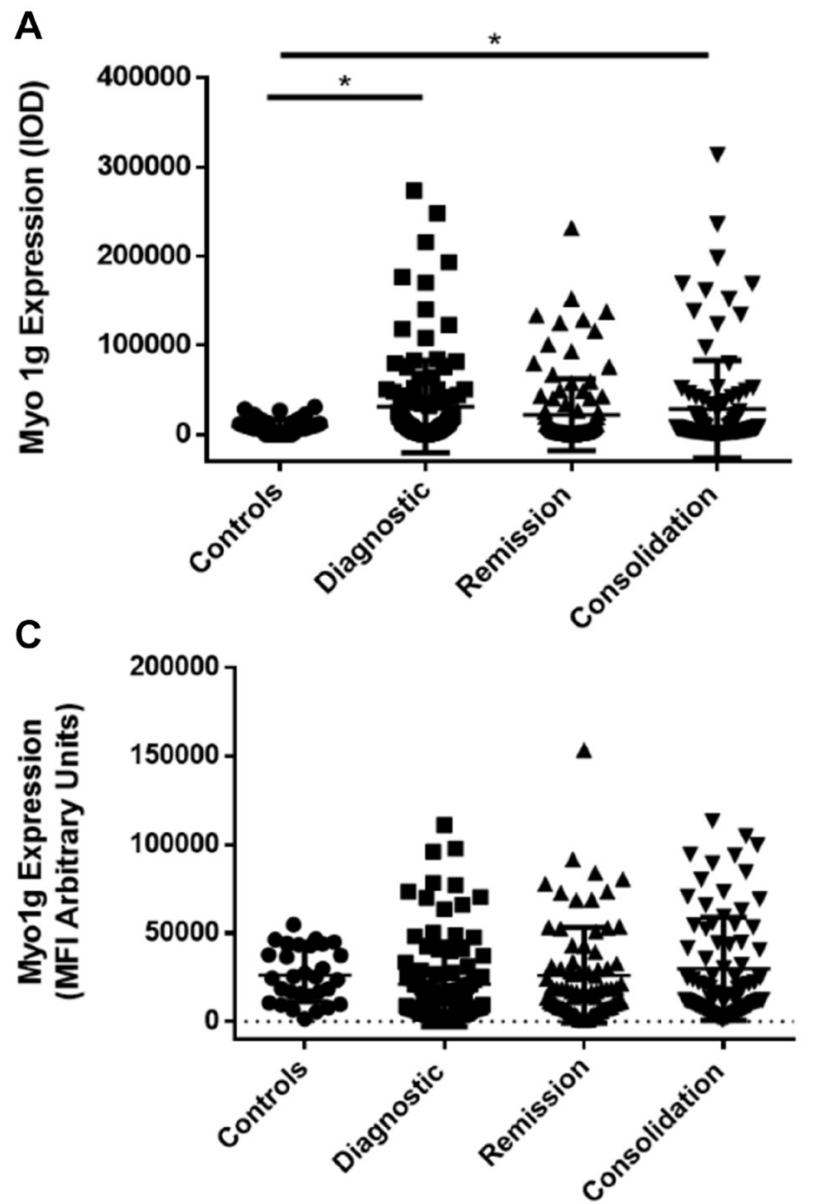

0.05 by qRT-PCR and ICQ respectively in patients with $t(9: 22)$, those patients classically are considered as very high risk because these leukemic cells are often resistant to therapy $[1,31,32]$. Therefore, will be interesting to evaluate te predictive value of Myo1g on this subset of patients. Myolg has been associated with cell migration and cell adhesion in $\mathrm{B}$ and $\mathrm{T}$ lymphocytes [16, 18, 33], and therefore is likely to be involved in leukemia infiltration, Importantly Myo1g deletion in mice has sown impairment of B cell migration [16] unfortunately, so far there are no studies over expressing Myolg in B cell lines or primary B cells demonstrating and increase in cell migration, that will be subject of future work in our lab, also over expressing Myolg and transferring those cells into nude mice to induce tumors and evaluate their ability to infiltrate different tissues will be an interesting approach. Myolg in normal conditions is exclusively expressed in hematopoietic cells however, Myo1g over

B

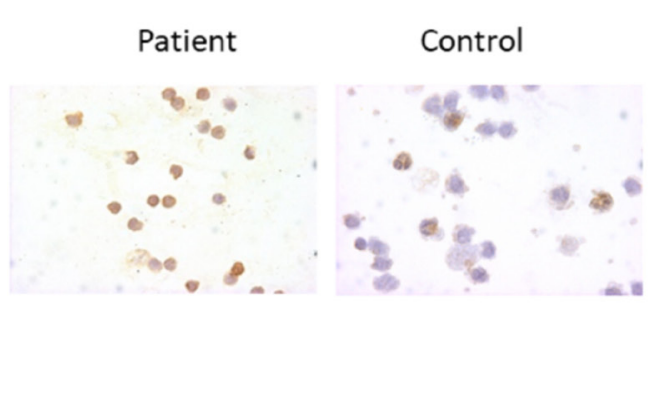

D
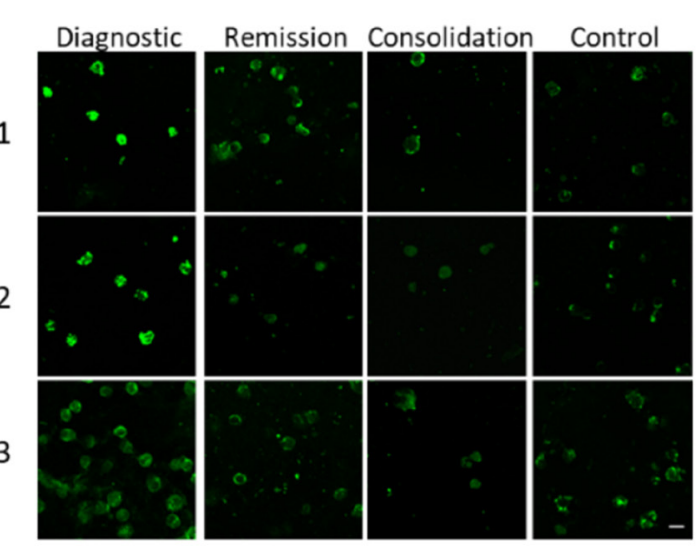

Figure 3: Myo1g over expression is conserved at protein level in acute lymphoblastic leukemia. (A) Intensity of Optical Density (IOD) of Myolg expression in PBMCs from control individuals $n=61$, patients at Diagnostic $n=117$, patients at remission $n=$ 105 and patients at consolidation $n=106$. Error bars represent mean $+/-\mathrm{SD}$, One-way ANOVA with Dunn's correction, ${ }^{*} P=0<0.05$. $(\mathbf{B})$ Representative images of Myo1g expression at diagnosis from a patient and a control individual. (C) Mean fluorescence intensity of Myo1g expression in PBMCs from patients with no relapse and with relapse at Diagnostic (D) $n=93, n=8$ respectively, patients at remission $n=$ 79 and $n=4$ respectively and patients at consolidation $n=77$ and $n=4$ respectively. Error bars represent mean $+/-\mathrm{SD}$, Unpaired $t$ test, ${ }^{*} P$ $=0<0.05$. (D) Representative confocal images of Myolg expression in 3 patients at diagnostic, remission and consolidation and 3 control individuals, bar represents $10 \mu \mathrm{m}$. 
expression (at mRNA level) is of bad prognostic in Renal Cancer in TCGA data, indeed high expression of Myolg indicates poor survival, similar trend was observed in our patients however, we didn't find significant differences in patient survival, but those patients who expressed high levels of Myolg (at protein and mRNA level) had in general poor survival. One intriguing feature of the Myolg over expression is that there was no significant decrease in the expression as the patients continued their treatment (consolidation); one possibility is that longer follow up is required and that Myolg expression will return to base line levels after long maintenance period. Myolg expression was similar in peripheral blood and bone marrow samples, this fact allowed us to continue the studies with PBMCs, a routine visit with a blood sample could help to evaluate the risk in the children because we detected elevated levels of Myo1g at diagnosis; therefore, maybe implementing regular test of Myolg in children at risk could help to detect cancerous cells at an early stage.

Class I myosins recently have gained interest in cancer research because some are over expressed and contribute to increase disease severity, for example Myolb in head and neck squamous cell carcinoma (HNSCC) promotes cell migration and lymph node metastasis, Myole in breast cancer promotes proliferation and tumor de differentiation $[24,30]$, some others are down regulated like Myola in colon cancer were seems to function as a tumor suppressor $[25,26]$. The analysis of Myolg expression in Leukemia showed that is a molecule that participates in the development of the pediatric ALL and together with the clinical characteristics could strengthen the initial diagnosis, although we know that the diagnosis of ALL is confirmed by the bone marrow aspirate, an extra indication in PBMCs could contribute to the correct patient stratification, further studies are necessary to define and to identify the underlying biochemical and functional mechanisms of the over expression of this myosin in the leukemic cells.

In summary, this study illustrates that Myolg expression might participate in the pathogenesis of the disease specially in high-risk patients of acute lymphoblastic leukemia and patients with translocation $\mathrm{t}(9: 22)$.

\section{MATERIALS AND METHODS}

\section{Patients and samples}

All human samples used in this study were authorized by written informed consent and approved by the Research Ethics and Bio safety Committee of the Hospital Infantil de Mexico, Federico Gómez and Hospital Pediátrico Moctezuma.

Peripheral blood was obtained from a cohort of 133 patients with acute lymphoblastic leukemia (ALL) and 10 samples of bone marrow from the same patients admitted from September 2015-July 2018. The clinical data are summarized in Table 1. Samples were obtained in EDTA tubes, at diagnosis and at two different stages of treatment: complete remission (in average Day 28) and one month after complete remission (consolidation). None of the patients had received treatment before diagnostic.

We included 60 no leukemia children as a control cohort. Informed consent was granted from all participants. The control children are in an age range of 1-15 years old, and during their participation in the study they did not present alterations in hematological values.

\section{Isolation of PBMCs}

Peripheral blood mononuclear cells were isolated by density gradient with Lymphoprep (Axis Shield), following the manufacturer's instructions. After this the samples were divided in two parts: one was stored in Trizol at $-80^{\circ} \mathrm{C}$ for mRNA extraction and the other was used for immunofluorescence and immunocytochemistry. Before staining, the cells were fixed in $4 \%$ paraformaldehyde on slides and then washed with PBS 1X.

\section{RNA isolation, reverse transcription and quantitative real-time PCR}

RNA was extracted from PBMCs using the RNeasy mini kit (QIAGEN), and reverse transcribed into cDNA using the Quantitect Reverse Transcription kit (QIAGEN). Myosin $1 \mathrm{G}$ expression was measured by quantitative PCR using the Agilent Mx3005 P thermocycler, Universal Probe Library (UPL) (Roche) with a specific primer set for myosin1G. Glyceraldehyde 3-phosphate dehydrogenase (GAPDH) gene was used as the internal control; for amplification we used the Light Cycler 480 (Roche) master mix, amplification was carried out at $95^{\circ} \mathrm{C} / 10$ $\mathrm{s}, 56-59^{\circ} \mathrm{C} / 30 \mathrm{~s}, 72^{\circ} \mathrm{C} / 11 \mathrm{~s}$ for 45 cycles. Fold Change values of gene expression were calculated with the $2^{-\Delta \Delta \mathrm{Ct}}$ method using the average from triplicate measurements.

\section{Immunofluorescence microscopy}

Cells were washed in $0.1 \%$ PBS / Tween 20 for 40 min. Samples were blocked with $2 \%$ Pig Serum (PS) and $0.5 \%$ Triton X-100 in a humidity chamber for $1 \mathrm{~h} 40 \mathrm{~min}$, then washed twice for $2 \mathrm{~min}$. Samples were incubated with the primary antibody for myosin $1 \mathrm{G}$, previously described [14] at room temperature overnight at a 1:100 dilution. After five washes with $0.1 \%$ PS in PBS/Tween 20, samples were incubated for $1 \mathrm{~h}$ at room temperature with goat anti-rabbit IgG Alexa 647 (Jackson ImmunoResearch) at 1:200 dilution; finally, cells were washed twice in PBS/ Tween 20. Coverslips were mounted on glass slides using vectashield mounting medium with DAPI (Vector Labs).

To image the stained cells, we used an Olympus FV1000 confocal microscope. We captured no less than 5 


\begin{tabular}{|c|c|}
\hline Patients ALL & 133 \\
\hline Age & Range ( 7 months to 16 years) \\
\hline Gender $n,(\%)$ & $52,(39) \mathrm{F} / 81$ (61) M \\
\hline Risk stratification (ALL patients) & HR 93/SR 39 \\
\hline \multirow[t]{4}{*}{ ALL Immunophenotype } & Pro - B 15 \\
\hline & Pre - B 87 \\
\hline & Т 9 \\
\hline & B 17 \\
\hline Deaths ALL $n,(\%)$ & $22,(16.5)$ \\
\hline Infiltration ALL $n,(\%)$ & $22,(16.5)$ \\
\hline Translocation 9:22 $n,(\%)$ & $5,(3.75)$ \\
\hline Relapse (\%) & $12,(9.02)$ \\
\hline No remission $n,(\%)$ & $15,(19.54)$ \\
\hline Controls & $n=61$ \\
\hline Age & Range ( $1-15$ years) \\
\hline
\end{tabular}

micrographs of the median plane of the cells at $60 \mathrm{X}$ using identical settings for each capture, making sure that no saturated pixels were detected. We quantified fluorescence intensity from 50 to 100 cells per patient at diagnosis and in the different phases of treatment (remission and consolidation). Images were analyzed using Fiji, Image J software (NIH); specifically, we draw a line in the periphery of each cell to measure the mean fluorescence intensity (MFI) in each cell. Results were expressed as the average of MFI for each patient using arbitrary units for the quantification.

\section{Immunocytochemistry}

The slides were incubated with antibodies for myosin $1 G$ [14] or normal rabbit IgG's to identify nonspecific binding (Normal Rabbit Serum, Santa Cruz Biotechnology). Cells were hydrated with PBS $1 \mathrm{X}$ for 5 minutes before antigen recovery with sodium citrate $(0.01 \mathrm{M}, \mathrm{pH} 6.0)$ for $15 \mathrm{~min}$ at $90^{\circ} \mathrm{C}$. Samples were washed twice with PBS $1 \mathrm{X}$. Endogenous peroxidase activity was eliminated with two washes of $15 \mathrm{~min}$ with methanol and $3 \%$ hydrogen peroxide. We used the same conditions for blocking and primary antibody incubation as in immunofluorescence assays, but anti-Myo1g was used at a 1:750 dilution. Cells were incubated with ImmPRESS anti-Rabbit-HRP (Vector Laboratories) for $10 \mathrm{~min}$ at room temperature, then washed with PBS and incubated with streptavidin for $10 \mathrm{~min}$. Color was developed incubating the sample with the chromogen Diaminobenzidine (Dako) for $1 \mathrm{~min}$; the reaction was stopped with water and samples were counterstained with hematoxylin for 1s Samples were then dehydrated under the following conditions: distilled water, $70 \%$ ethanol, $90 \%$ ethanol, $100 \%$ ethanol and xylene; finally, glass slides were covered with resin. Cells were visualized using an
Olympus BX-40 microscope, intensity from 50 to 100 cells per patient at diagnosis and in the different phases of treatment was measured to obtain the IOD (Intensity of Optical Density), using the software Image-Pro Plus V. 6.0. The brown colour staining of the cellular periphery was quantified and all values from one patient per treatment phase were averaged. Results were expressed as the average of the quantified pixels (IOD) in each patient.

\section{Statistical analyses}

Statistical tests were performed with GraphPad Prism Software (version 6.01, La Jolla, CA, USA). Comparison between 2 groups was evaluated by unpaired $t$-test or Mann Whitney test (for variables with no normal distribution). For multiple comparisons, we performed one-way ANOVA and Kruskal-Wallis with Dunn's correction for variables with non-normal distribution. The suitability of Myolg as a biomarker that discriminates ALL patients and normal subjects was evaluated by calculating the area under the curve (AUC) using SPSS version 20. Kaplan Meier analysis was performed to estimate the survival function from lifetime data. Statistical significance was assessed by Log Rank test. Unless otherwise stated results are shown mean $+/-$ SD. $P$ values $<0.05$ were considered as statistically significant.

\section{Author contributions}

LEA, JAC, YGG, GAA, JDV performed experiments and analyzed data, LRC, ASA, SHY provided critical reagents and revised the manuscript, DOR, JTN provided clinical samples, GPL conceived the project, acquired funding, analyzed data and drafted the manuscript with input from all the authors. 


\section{ACKNOWLEDGMENTS}

We thank to all patients and their guardians for accepting to participate in this study, we also thank to Dra. Elisa Dorantes, Dr. Felix Gaytan and Dr. Ivan Castorena for their help at the beginning of our study and to the residents who rotated in the Hemato/oncology department of both hospitals and helped with the obtention of samples.

\section{CONFLICTS OF INTEREST}

Authors have no conflicts of interest to declare.

\section{FUNDING}

This work was funded by Federal Funds from the Hospital Infantil de México, Federico Gómez through grants HIM 2013/061 SSA 1090 And HIM 2017/014 SSA 1308.

\section{REFERENCES}

1. Tasian SK, Loh ML, Hunger SP. Childhood acute lymphoblastic leukemia: Integrating genomics into therapy. Cancer. 2015; 121:3577-90. https://doi.org/10.1002/ cncr.29573. [PubMed]

2. Pérez-Saldivar ML, Fajardo-Gutiérrez A, Bernáldez-Ríos R, Martínez-Avalos A, Medina-Sanson A, EspinosaHernández L, Flores-Chapa Jde D, Amador-Sánchez R, Peñaloza-González JG, Alvarez-Rodríguez FJ, BoleaMurga V, Flores-Lujano J, Rodríguez-Zepeda Mdel C, et al. Childhood acute leukemias are frequent in Mexico City: descriptive epidemiology. BMC Cancer. 2011; 11:355. https://doi.org/10.1186/1471-2407-11-355. [PubMed]

3. Miller KD, Nogueira L, Mariotto AB, Rowland JH, Yabroff KR, Alfano CM, Jemal A, Kramer JL, Siegel RL. Cancer treatment and survivorship statistics, 2019. CA Cancer J Clin. 2019; 69:363-85. https://doi.org/10.3322/caac.21565. [PubMed]

4. Siegel RL, Miller KD, Jemal A. Cancer statistics, 2019. CA Cancer J Clin. 2019; 69:7-34. https://doi.org/10.3322/ caac.21551. [PubMed]

5. Ward E, DeSantis C, Robbins A, Kohler B, Jemal A. Childhood and adolescent cancer statistics, 2014. CA Cancer J Clin. 2014; 64:83-103. https://doi.org/10.3322/ caac.21219. [PubMed]

6. Zhang X, Wu H, Fan H, Su B, Zhang G, Dong L. Clinical characteristics and prognosis of pediatric patients with $\mathrm{B}$ cell acute lymphoblastic leukemia relapse. Oncol Lett. 2018; 16:2929-34. https://doi.org/10.3892/ol.2018.8974. [PubMed]

7. Jost TR, Borga C, Radaelli E, Romagnani A, Perruzza L, Omodho L, Cazzaniga G, Biondi A, Indraccolo S, Thelen M, Te Kronnie G, Grassi F. Role of CXCR4-mediated bone marrow colonization in CNS infiltration by $\mathrm{T}$ cell acute lymphoblastic leukemia. J Leukoc Biol. 2016; 99:1077-87. https://doi.org/10.1189/jlb.5MA0915-394R. [PubMed]

8. Izdebska M, Zielińska W, Grzanka D, Gagat M. The Role of Actin Dynamics and Actin-Binding Proteins Expression in Epithelial-to-Mesenchymal Transition and Its Association with Cancer Progression and Evaluation of Possible Therapeutic Targets. Biomed Res Int. 2018; 2018:4578373. https://doi.org/10.1155/2018/4578373. [PubMed]

9. Parsons JT, Horwitz AR, Schwartz MA. Cell adhesion: integrating cytoskeletal dynamics and cellular tension. Nat Rev Mol Cell Biol. 2010; 11:633-43. https://doi. org/10.1038/nrm2957. [PubMed]

10. Osherov N, May GS. In vivo function of class I myosins. Cell Motil Cytoskeleton. 2000; 47:163-73. https:// doi.org/10.1002/1097-0169(200011)47:3<163::AIDCM1>3.0.CO;2-U. [PubMed]

11. McIntosh BB, Ostap EM. Myosin-I molecular motors at a glance. J Cell Sci. 2016; 129:2689-95. https://doi. org/10.1242/jes.186403. [PubMed]

12. Liu KC, Cheney RE. Myosins in cell junctions. Bioarchitecture. 2012; 2:158-70. https://doi.org/10.4161/ bioa.21791. [PubMed]

13. McConnell RE, Tyska MJ. Leveraging the membrane cytoskeleton interface with myosin-1. Trends Cell Biol. 2010; 20:418-26. https://doi.org/10.1016/j.tcb.2010.04.004. [PubMed]

14. Patino-Lopez G, Aravind L, Dong X, Kruhlak MJ, Ostap EM, Shaw S. Myosin $1 \mathrm{G}$ is an abundant class I myosin in lymphocytes whose localization at the plasma membrane depends on its ancient divergent pleckstrin homology $(\mathrm{PH})$ domain (Myo1PH). J Biol Chem. 2010; 285:8675-86. https://doi.org/10.1074/jbc.M109.086959. [PubMed]

15. Olety B, Wälte $M$, Honnert U, Schillers H, Bähler M. Myosin $1 \mathrm{G}$ (Myo1G) is a haematopoietic specific myosin that localises to the plasma membrane and regulates cell elasticity. FEBS Lett. 2010; 584:493-99. https://doi. org/10.1016/i.febslet.2009.11.096. [PubMed]

16. Maravillas-Montero JL, López-Ortega O, Patiño-López G, Santos-Argumedo L. Myosin 1g regulates cytoskeleton plasticity, cell migration, exocytosis, and endocytosis in B lymphocytes. Eur J Immunol. 2014; 44:877-86. https://doi. org/10.1002/eji.201343873. [PubMed]

17. Santos-Argumedo L, Maravillas-Montero JL, López-Ortega O. Class I myosins in B-cell physiology: functions in spreading, immune synapses, motility, and vesicular traffic. Immunol Rev. 2013; 256:190-202. https://doi.org/10.1111/ imr.12105. [PubMed]

18. Gérard A, Patino-Lopez G, Beemiller P, Nambiar R, BenAissa K, Liu Y, Totah FJ, Tyska MJ, Shaw S, Krummel MF. Detection of rare antigen-presenting cells through $\mathrm{T}$ cell-intrinsic meandering motility, mediated by Myo1g. Cell. 2014; 158:492-505. https://doi.org/10.1016/j. cell.2014.05.044. [PubMed] 
19. Maravillas-Montero JL, Gillespie PG, Patiño-López G, Shaw S, Santos-Argumedo L. Myosin 1c participates in B cell cytoskeleton rearrangements, is recruited to the immunologic synapse, and contributes to antigen presentation. J Immunol. 2011; 187:3053-63. https://doi. org/10.4049/jimmunol.1004018. [PubMed]

20. Ouderkirk JL, Krendel M. Non-muscle myosins in tumor progression, cancer cell invasion, and metastasis. Cytoskeleton (Hoboken). 2014; 71:447-63. https://doi. org $/ 10.1002 / \mathrm{cm} .21187$. [PubMed]

21. Peckham M. How myosin organization of the actin cytoskeleton contributes to the cancer phenotype. Biochem Soc Trans. 2016; 44:1026-34. https://doi.org/10.1042/ BST20160034. [PubMed]

22. Li YR, Yang WX. Myosins as fundamental components during tumorigenesis: diverse and indispensable. Oncotarget. 2016; 7:46785-812. https://doi.org/10.18632/ oncotarget.8800. [PubMed]

23. Ouderkirk JL, Krendel M. Myosin 1e is a component of the invadosome core that contributes to regulation of invadosome dynamics. Exp Cell Res. 2014; 322:265-76. https://doi.org/10.1016/j.yexcr.2014.01.015. [PubMed]

24. Ouderkirk-Pecone JL, Goreczny GJ, Chase SE, Tatum AH, Turner CE, Krendel M. Myosin 1e promotes breast cancer malignancy by enhancing tumor cell proliferation and stimulating tumor cell de-differentiation. Oncotarget. 2016; 7:46419-32. https://doi.org/10.18632/oncotarget.10139. [PubMed]

25. Mazzolini R, Dopeso H, Mateo-Lozano S, Chang W, Rodrigues P, Bazzocco S, Alazzouzi H, Landolfi S, Hernández-Losa J, Andretta E, Alhopuro P, Espín E, Armengol M, et al. Brush border myosin Ia has tumor suppressor activity in the intestine. Proc Natl Acad Sci U S A. 2012; 109:1530-35. https://doi.org/10.1073/ pnas.1108411109. [PubMed]

26. Mazzolini R, Rodrigues P, Bazzocco S, Dopeso H, Ferreira AM, Mateo-Lozano S, Andretta E, Woerner SM, Alazzouzi H, Landolfi S, Hernandez-Losa J, Macaya I, Suzuki H, et al. Brush border myosin Ia inactivation in gastric but not endometrial tumors. Int J Cancer. 2013; 132:1790-99. https://doi.org/10.1002/ijc.27856. [PubMed]

27. Ohmura G, Tsujikawa T, Yaguchi T, Kawamura N, Mikami S, Sugiyama J, Nakamura K, Kobayashi A, Iwata T, Nakano H, Shimada T, Hisa Y, Kawakami Y. Aberrant Myosin 1b
Expression Promotes Cell Migration and Lymph Node Metastasis of HNSCC. Mol Cancer Res. 2015; 13:72131. https://doi.org/10.1158/1541-7786.MCR-14-0410. [PubMed]

28. Meyer LH, Eckhoff SM, Queudeville M, Kraus JM, Giordan M, Stursberg J, Zangrando A, Vendramini E, Möricke A, Zimmermann M, Schrauder A, Lahr G, Holzmann K, et al. Early relapse in ALL is identified by time to leukemia in NOD/SCID mice and is characterized by a gene signature involving survival pathways. Cancer Cell. 2011; 19:206-17. https://doi.org/10.1016/j.ccr.2010.11.014. [PubMed]

29. Portell CA, Advani AS. Novel targeted therapies in acute lymphoblastic leukemia. Leuk Lymphoma. 2014; 55:73748. https://doi.org/10.3109/10428194.2013.823493. [PubMed]

30. Zhang HR, Lai SY, Huang LJ, Zhang ZF, Liu J, Zheng SR, Ding K, Bai X, Zhou JY. Myosin $1 \mathrm{~b}$ promotes cell proliferation, migration, and invasion in cervical cancer. Gynecol Oncol. 2018; 149:188-97. https://doi. org/10.1016/i.ygyno.2018.01.024. [PubMed]

31. Shin J, Lee NY, Kim S, Lee JW, Jang PS, Chung NG, Cho B. Outcome and prognostic factors of children with Philadelphia chromosome-positive acute lymphoblastic leukemia treated with imatinib followed by allogeneic hematopoietic cell transplantation in first remission. Blood Res. 2019; 54:45-51. https://doi.org/10.5045/ br.2019.54.1.45. [PubMed]

32. Schultz KR, Carroll A, Heerema NA, Bowman WP, Aledo A, Slayton WB, Sather H, Devidas M, Zheng HW, Davies SM, Gaynon PS, Trigg M, Rutledge R, et al, and Children's Oncology Group. Long-term follow-up of imatinib in pediatric Philadelphia chromosome-positive acute lymphoblastic leukemia: Children's Oncology Group study AALL0031. Leukemia. 2014; 28:1467-71. https://doi. org/10.1038/leu.2014.30. [PubMed]

33. López-Ortega O, Santos-Argumedo L. Myosin 1g Contributes to CD44 Adhesion Protein and Lipid Rafts Recycling and Controls CD44 Capping and Cell Migration in B Lymphocytes. Front Immunol. 2017; 8:1731. https:// doi.org/10.3389/fimmu.2017.01731. [PubMed] 